\title{
El uso de metáforas en el discurso de construcción científica en estudiantes de educación preescolar
}

\section{The use of metaphors in the scientific construction discourse of preschool students}

DOI: https://doi.org/10.32870/dse.v0i20.598

\author{
Vanessa de Alba Villaseñor*
}

\begin{abstract}
Resumen
La línea de investigación sobre modelización respecto a la ciencia que se hace en preescolar representa un campo de conocimiento poco explorado. En este trabajo se pretende analizar los procesos de interacción social en el aula, desarrollados por un grupo de estudiantes de 5 y 6 años de edad, durante una secuencia didáctica relacionada con el modelo de ser vivo enfocado en el sistema circulatorio.

Candela (1999) señala que para comprender el discurso explicativo es necesario partir de elementos culturales de los sujetos, hacerse parte del grupo social e identificar las estrategias comunicativas que permitan entender los procesos de elaboración conceptual, a través de un análisis de interacciones con enfoque etnográfico.

La estrategia comunicativa que se analiza en este trabajo es el uso de metáforas. Lakoff y Johnson (1995) clasifican dos grupos de metáforas: estructurales y empíricas. Los resultados muestran que los alumnos de preescolar incluían elementos de su contexto físico, es decir, metáforas empíricas, para explicar el proceso de circulación sanguínea, así como características de los órganos involucrados.
\end{abstract}

Palabras clave: modelización científica - educación preescolar - análisis de discurso -metáforas.

\begin{abstract}
The line of research on modeling regarding science in preschool is a seldom-explored field of knowledge. This paper aims to analyze the processes of social interaction in the classroom developed by a group of five- and six-year old students during a didactic sequence related to the model of a living being and focused on the circulatory system.

Candela (1999) points out that in order to understand the explanatory discourse, it is necessary to start from the cultural elements of the subjects, become part of the social group, and identify the communicative strategies that allow us to understand the processes of conceptual elaboration, through an analysis of interactions with an ethnographic approach.
\end{abstract}

\footnotetext{
* Doctora en Investigación Educativa Aplicada. Universidad Marista de Guadalajara. México. Líneas de investigación: Enseñanza de las ciencias, Pensamiento científico. vanessa.dealba@umg.edu.mx
} 
The communicative strategy analyzed in this work is the use of metaphors. Lakoff and Johnson (1995) classify two groups of metaphors: structural and empirical. The results show that preschool students included elements of their physical context, that is, empirical metaphors, to explain the process of blood circulation, as well as the characteristics of the organs involved.

Keywords: scientific modeling - preschool education - discourse analysis - metaphors.

\section{Introducción}

Aprender ciencias en educación preescolar, para el estudiante implica vivenciar los procesos de construcción de significados a través de una interacción social de organización conceptual, y sus ideas iniciales desempeñan un papel de vital importancia (Canedo, Castelló, García, Gómez y Morales, 2012). En este sentido, la ciencia escolar (Gómez, 2007) puede ser entendida como los procedimientos por medio de los cuales el niño comprende el mundo, entre los más importantes está la experimentación y el diálogo.

Comprender cómo los estudiantes de educación preescolar hacen ciencia, es un tema que comienza a cobrar relevancia alrededor del mundo. Dos trabajos de investigación rescatan los procesos de adquisición de saberes científicos con estudiantes de 3 a 6 años de edad (CruzGuzmán, García y Criado, 2017; Howitt et al., 2011) desde una perspectiva cualitativa empleando técnicas descriptivas.

Cruz-Guzmán, García y Criado (2017), y Howitt, Lewis y Upson (2011) realizaron diseños didácticos basados en preguntas-predicción, demostrando que las hipótesis de los estudiantes se incrementaron, así como la capacidad de experimentación, y se dieron cuenta de la importancia de rescatar el contexto a través de narrativas. Sus resultados dan cuenta de la capacidad que tienen los alumnos de sustentar explicaciones en observaciones y registros; por ello, consideran relevante brindar variadas oportunidades de este tipo al hacer ciencia escolar.

Por otro lado, Gómez-Motilla y Ruiz-Gallardo (2016), en un estudio que buscaba medir la actitud hacia la ciencia en niños de 3 y 4 años, probaron que experimentar con los sentidos estimula la curiosidad y la construcción de explicaciones causales de fenómenos, favoreciendo la actitud y el interés científico.

Ahora, con respecto a la construcción de modelos mentales, se encontraron tres estudios sobre interpretación de dibujos de los niños, con los que describen y en algunos casos explican cómo suceden varios fenómenos, como en el caso de las especies que viven en el desierto (Ahi, 2016). Oğuzhan, Altan y Ahi (2013) describen el aula de ciencias ideal desde las concepciones de los estudiantes.

Por su parte, Saçkes (2015) analiza las explicaciones sobre el día y la noche, y las categoriza en tres tipos de modelos: el inicial, que incluye elementos del fenómeno con algunas descripciones; el sintético, en el que se consideran relaciones entre sus componentes; y por último el

Diálo@os

Sobre Educación año 11 | número 20 | enero-junio 2020 | ISSN 2007-2171 
científico, en el que, además de lo anterior, se presentan explicaciones causales o de función. Desde el enfoque de modelización a través de secuencias de experimentación, Mazas, Gil, Martínez, Hervas y Muñoz (2018) diseñaron el desarrollo de destrezas de observación, comparación, formulación de hipótesis y nuevas preguntas, así como explicaciones de comportamiento acerca de dos fenómenos: el aire y el agua.

\section{Aprender ciencias en preescolar en el ámbito nacional}

En México es reciente el interés por conocer los procesos científicos de los alumnos de preescolar. Gómez, García y García (2013) realizaron un estado del conocimiento que comprende desde el año 2002 hasta 2011. Localizaron 199 trabajos en educación básica — de los cuales diez se realizaron en preescolar - con el propósito de demostrar la capacidad que tienen los alumnos de comprender las ciencias mediante secuencias didácticas acerca de física y biología. La perspectiva teórico-metodológica privilegiada fue la cognitiva sociocultural. Tres de estas investigaciones corresponden al enfoque de modelización en las que se construyen modelos teóricos escolares de ser vivo; sus resultados dan cuenta de la elaboración de significados consensuados por los alumnos.

Con la certeza de que los estudiantes entre 5 y 6 años de edad son capaces de aprender ciencias, Canedo-lbarra et al. (2012) analizaron los procesos de modificación de concepciones iniciales con respecto a los seres vivos y no vivos. Ellos encontraron dos tipos de patrones de aprendizaje; uno que se consideró en transición ya que en las explicaciones prevalecen las caracterizaciones animistas, aunque se agregan propiedades biológicas; $y$ otro que se considera científico, donde la diferencia entre seres vivos y no vivos es sustentada en su totalidad en propiedades y procesos biológicos.

Ahora bien, con la presente investigación se intenta sumar a la búsqueda por comprender los procesos científicos de los alumnos de educación preescolar, específicamente en el uso de recursos explicativos para la construcción de significados consensuados que permitan generar modelos teóricos escolares.

\section{El lenguaje científico}

La enseñanza de la ciencia siempre ha estado vinculada con el lenguaje (Espinet, Izquierdo, Bonfil y Ramos, 2012). A mediados del siglo XX, desde una postura puramente semántica, se focalizaba la mirada en el significado de las teorías y se usaban los modelos como proyecciones de la realidad. En este sentido, el modelo era considerado una representación simbólica que permitía pensar y actuar sobre el fenómeno que se intentaba conocer, y así, la ciencia que se hacía al modelizar permitía transformar los hechos cotidianos en hechos científicos, y el lenguaje cotidiano en lenguaje científico. 
Con respecto al lenguaje, en las últimas décadas han surgido tres importantes perspectivas: a) el lenguaje como un medio, b) el lenguaje como teoría y acción, y c) el lenguaje como interacción.

En la perspectiva del lenguaje como medio, este es considerado como una representación simbólica cargada de significados que se construyen en la mente desde una estructura de pensamiento lógico; es el vehículo para dar a conocer un proceso y su elaboración mental. Por otra parte, en la perspectiva del lenguaje como teoría y acción, el lenguaje es una herramienta que permite razonar, es decir, al mismo tiempo que se interpretan los fenómenos, se desarrollan habilidades lingüísticas cognitivas a través de un proceso dialéctico reflexivo que modifica lo que se dice y lo que se piensa, y su representación. Y por último, en la perspectiva del lenguaje como interacción, se reconoce la influencia social en la actividad científica y en la construcción de significados, se valida la ciencia que se hace en la escuela, así como los modelos construidos llamándolos modelos teóricos escolares, y resalta la importancia de la experimentación para el desarrollo de lenguaje y pensamiento, cada vez más científico (Espinet et al., 2012).

En esta perspectiva del lenguaje como interacción se inserta este trabajo de investigación, en el que fue relevante el acompañamiento de una docente de preescolar, la experimentación con materiales que permitieron vivenciar el fenómeno y, donde la elaboración del modelo teórico escolar se configuró por medio del intercambio verbal, principalmente, entre estudiantes.

\section{El enfoque de modelización}

En este apartado se inicia por definir qué se entiende por modelo, modelización y modelo teórico escolar. Modelo (Giere, 1988) es una unidad abstracta, un elemento intermedio que permite representar el vínculo entre la teoría que se intenta comprender y el significado que representa para los sujetos. Una forma de hacer ciencia escolar es la construcción de modelos.

La modelización es un enfoque de enseñanza que constituye todo un lenguaje científico (Izquierdo, Espinet, Puyol y Sanmartí, 1999). Implica un proceso que inicia con el establecimiento de un modelo teórico de referencia, continúa con la identificación de saberes previos de los estudiantes, la experimentación con variedad de materiales que les permitan vivenciar el fenómeno y la discusión con pares, de tal manera que puedan reconstruir hechos del mundo. Para Gómez (2009), la experimentación es el elemento fundamental de la modelización y constituye la base para la elaboración de explicaciones.

En este sentido, el proceso de modelización con estudiantes de educación preescolar se trata de comprender y apropiarse de elementos clave del modelo teórico de referencia; no se buscan construcciones científicas eruditas sino modificaciones en sus concepciones iniciales. Por tanto, es pertinente llamar a dicha construcción modelo teórico escolar (Canedo-lbarra et al., 2012). 
El modelo teórico escolar se trata de una creación original congruente con el modelo teórico erudito de referencia (Gómez, 2005). Surge en un contexto determinado y permite al alumno comprender el fenómeno. Tiene su fundamento en el enfoque cognitivo sociocultural de Vygotsky (Izquierdo et al., 1999), se construye a través de la experimentación y la interacción con compañeros de clase; de este modo, el lenguaje desempeña un papel fundamental.

De manera concreta, dentro del enfoque de modelización (Izquierdo et al., 1999), podemos entender el modelo teórico escolar como el medio que facilita comprender el mundo real cuyos elementos clave son: la teoría, el modelo y la realidad.

En este sentido, el proceso de construcción del modelo teórico escolar posee una función mediadora, ya que busca conectar los hechos del mundo (fenómenos) con los hechos científicos (modelos teóricos). Adúriz-Bravo, Gómez, Márquez y Sanmartí (2005) llaman a esta función mediadora potencia analógica, puesto que implica crear analogías o metáforas que ayuden a establecer relaciones de semejanza como recurso explicativo.

Con estos planteamientos como marco teórico, se estableció el objetivo de analizar, a través de la interacción en el aula, los procesos de modelización científica desarrollados en un grupo de estudiantes de educación preescolar, durante una secuencia didáctica relacionada con el modelo de ser vivo, específicamente, el sistema circulatorio.

A partir de ello, las preguntas de investigación se orientaron a identificar: ¿Cuáles son las características que definen los procesos de modelización científica al trabajar con el modelo del sistema circulatorio en educación preescolar? y ¿Qué tipo de modelo del sistema circulatorio construyen los estudiantes.

\section{Modelo de ser vivo y modelo del sistema circulatorio}

Entender la composición y desarrollo de distintos procesos en el ser vivo es un tema ampliamente estudiado y modelizado de manera erudita (García, 2005). Las cuatro funciones que integran el modelo de ser vivo y lo distinguen de los objetos son: nutrición, relación, reproducción y de estructura celular.

Para este trabajo se estableció un modelo teórico de referencia basado en los planteamientos de Gutiérrez (2015). Dicho modelo está enfocado al sistema circulatorio del cuerpo humano, congruente con el modelo de ser vivo (véase figura 1). En él se consideran elementos de estructura, función y enfermedad. En este caso solo se tomó en cuenta lo referente a estructura y función. 
Figura 1. Modelo teórico de referencia focalizado en el sistema circulatorio del cuerpo humano basado en Gutiérrez (2015).

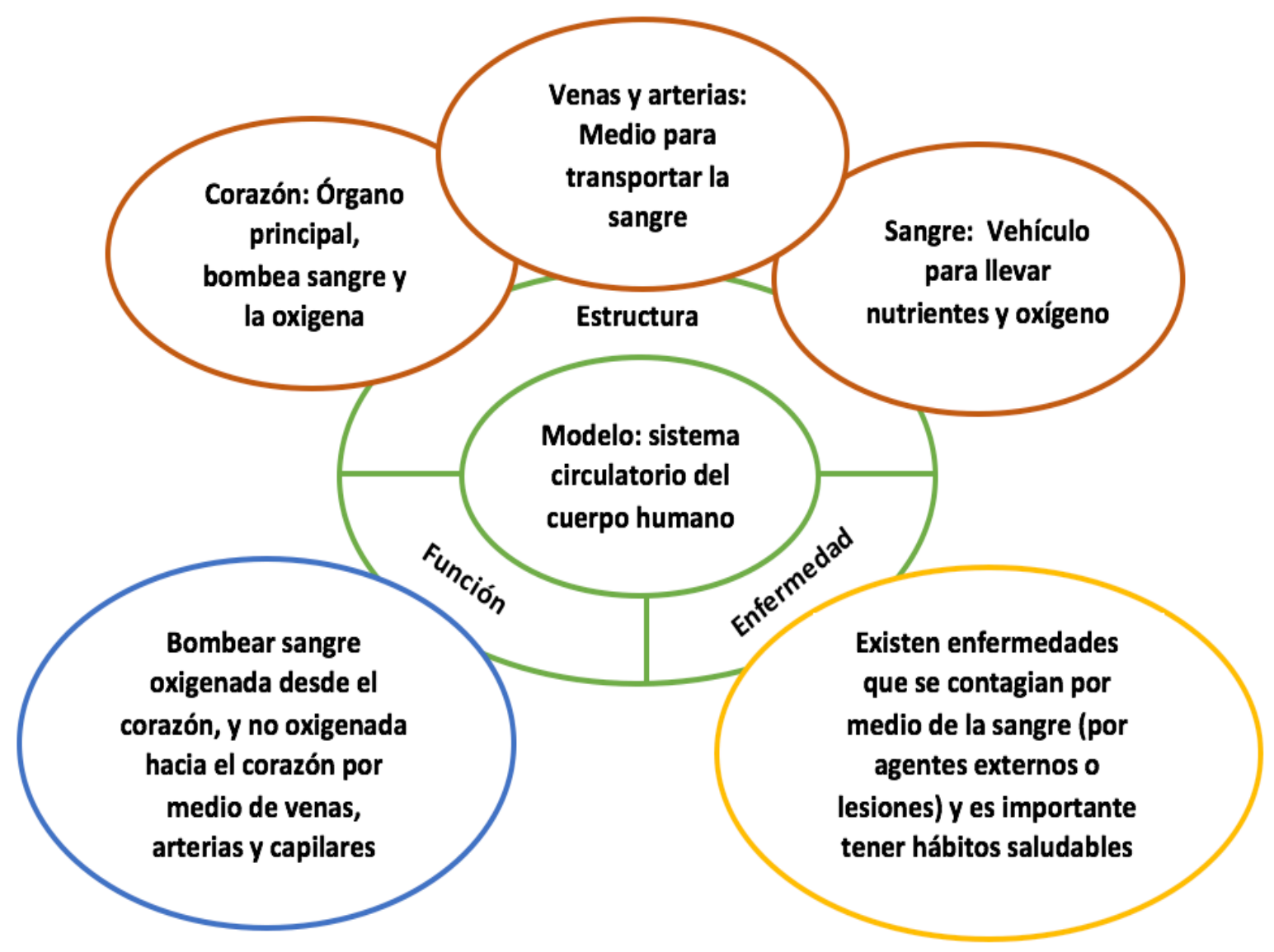

\section{Metodología}

El desarrollo de la investigación consistió en la aplicación de una secuencia didáctica con 21 estudiantes entre los 5 y 6 años de edad en un Jardín de Niños del municipio de Ixtlahuacán de los Membrillos, Jalisco, durante el ciclo escolar 2015-2016. El grupo estaba conformado por 12 niños y 9 niñas. Los datos se obtuvieron por medio de videograbaciones de clase y dibujos que se interpretaron junto con los estudiantes por medio de entrevistas.

Se buscaba comprender los procesos de modelización científica relacionados con la construcción de significados de los alumnos, se consideró entonces que el instrumento principal debía ser el lenguaje.

Por ello, la decisión metodológica se orientó a la realización de un análisis de interacciones desde el enfoque etnográfico, que tomara en cuenta la participación activa de la investigadora - autora de este artículo- como parte del equipo de enseñanza, con apoyo de la profesora de 
grupo, y los intercambios comunicativos que constituyen el sistema de mediación teórica (Candela, 1999), es decir, los procesos de interacción oral que llevan a la elaboración conceptual.

En este caso, los presupuestos del enfoque de modelización constituyeron el sistema de mediación teórica con el que se buscaba elaborar consensuadamente conceptos, poniendo en juego saberes previos, experimentaciones e intercambios comunicativos, con la intención de ajustar sus concepciones agregando elementos, estableciendo nuevos enlaces, reglas y jerarquizaciones (Canedo-lbarra et al., 2012).

Los presupuestos de la modelización consideran tres relaciones principales: la primera con el mundo físico, mediante experimentaciones; la segunda entre participantes, por medio de diálogos que permiten desarrollar explicaciones; y la tercera, con el conocimiento científico, para lo cual se toma un modelo teórico de referencia (Acher, Arcà y Sanmartí, 2007).

\section{Secuencia didáctica}

Se desarrolló una secuencia didáctica tomando como base, por un lado, los planteamientos del Programa de Estudios (SEP, 2012), que se fundamenta en un enfoque reflexivo en el que se invita a la experimentación y estimula la observación y la descripción, y por el otro, los elementos y relaciones del modelo teórico de ser vivo focalizado en el sistema circulatorio (figura 1), mediante cinco temas, los cuales se desarrollaron durante 14 jornadas de trabajo. De manera general, se puede apreciar en la siguiente tabla la distribución de temas, objetivos y duración.

Tabla 1. Secuencia didáctica del modelo de ser vivo focalizado en el sistema circulatorio

\begin{tabular}{|l|l|l|}
\hline Tema & Objetivo & No. de jornadas \\
\hline $\begin{array}{l}\text { El cuerpo por fuera } \\
\text { y por dentro }\end{array}$ & $\begin{array}{l}\text { Describir características y comparar el cuerpo } \\
\text { humano con el de animales después de exami- } \\
\text { narlos. }\end{array}$ & 4 (12 hrs.) \\
\hline $\begin{array}{l}\text { Experiencias con la } \\
\text { sangre }\end{array}$ & $\begin{array}{l}\text { Expresar con ideas propias cómo ocurren fenó- } \\
\text { menos con la sangre. } \\
\text { Observar con los sentidos. }\end{array}$ & 4 (12 hrs.) \\
\hline $\begin{array}{l}\text { ¿Dónde están las } \\
\text { venas? }\end{array}$ & $\begin{array}{l}\text { Identificar similitudes y diferencias entre venas } \\
\text { de animales y nervaduras de plantas. }\end{array}$ & 2 (6 hrs.) \\
\hline $\begin{array}{l}\text { ¿Cómo es que } \\
\text { funciona el corazón? }\end{array}$ & $\begin{array}{l}\text { Elaborar explicaciones propias acerca de los } \\
\text { procesos que vinculan las venas, la sangre y el } \\
\text { corazón. }\end{array}$ & 2 (6 hrs.) \\
\hline Termoregulación & $\begin{array}{l}\text { Comunicar resultados sobre la función de la san- } \\
\text { gre para regular la temperatura de los órganos } \\
\text { internos. }\end{array}$ & 2 (6 hrs.) \\
\hline
\end{tabular}

En este artículo se presentan los intercambios comunicativos del tema “Dónde están las venas?", debido a que lo que se pretende analizar es uno de los procesos de modelización cien- 
tífica: el uso de metáforas (Wigdorovitz, 2014) durante la elaboración de explicaciones, y es en dicho tema donde se presentaron en mayor proporción.

Como se puede apreciar en la tabla 1, el objetivo de este tema era establecer comparaciones entre distintos elementos, como corazones de pollo, nervaduras de apio y lechuga, con sus saberes previos respecto a las venas del cuerpo humano. Las actividades consistieron principalmente en la observación directa de los elementos ya mencionados y con instrumentos como lupas, para luego, a través de diálogos en pequeños equipos (4 o 5 integrantes), construir explicaciones utilizando registros gráficos, que posteriormente expusieron ante sus compañeros.

\section{Sistema de mediación teórica}

Los procesos sociales de elaboración conceptual constituyen los sistemas de mediación teórica (Candela, 1999). En este caso nos referimos a las acciones de modelización como el rescate de ideas previas, experimentación por medio de los sentidos y elaboración colectiva de explicaciones utilizando recursos propios.

Para comprender cómo se elaboran los conceptos en el aula es necesario identificar las diferentes formas de interacción que tienen relación con lo que se dice, cómo se dice, dónde se dice y las acciones previas que dan pie a la construcción social de un significado en particular; se trata de reconocer las conexiones en el lenguaje con el que se desarrollan explicaciones.

Una de esas conexiones, que se emplea con frecuencia en la ciencia que se hace en preescolar, es el uso de metáforas; estas constituyen la expresión verbal de una operación mental (Wigdorovitz, 2014), es decir, se relacionan con las maneras de pensar, orientan la comprensión y permiten construir colectivamente un significado. Lemke (1990) las describe como una relación de equivalencia para explicar un término o concepto científico empleando vocabulario común. En sintonía con lo anterior, las metáforas sirven para entender y experimentar una idea en términos de algo conocido (Lakoff y Johnson, 1995).

Las metáforas se clasifican en dos grandes grupos, estructurales y empíricas (Lakoff y Johnson, 1995). Las estructurales se mueven en el mundo de lo abstracto, buscan organizar una idea en términos de otra y su significado tiene sentido en un contexto determinado, un ejemplo de ello es la expresión: "el tiempo es dinero", se trata de una comparación entre dos elementos intangibles que pueden ser comprendidos en ciertas sociedades, pero no en todas. Las metáforas empíricas, por el contrario, tienen una base física y cultural, permiten organizar conceptos estableciendo relaciones con elementos del entorno y nos remiten a la experiencia; un ejemplo es la oración: "está en la cima del éxito", en este caso se usa como referencia un elemento de orientación espacial.

En el caso de los discursos analizados de los alumnos de preescolar, únicamente se encontraron metáforas empíricas; por esta razón solo se presenta en la siguiente tabla una caracterización de los tres tipos de metáforas que pertenecen a este grupo, fundamentada en Lakoff y Johnson (1995).

Diólo@os sobre Educación año 11 | número 20 | enero-junio 2020 | ISSN 2007-2171 
El uso de metáforas en el discurso de construcción científica en estudiantes de educación preescolar

Tabla 2. Caracterización de metáforas empíricas basada en Lakoff y Johnson (1995)

\begin{tabular}{|l|l|}
\hline Tipo & Descripción \\
\hline Orientacional & $\begin{array}{l}\text { Se usan elementos de ubicación espacial de referencia a través de tér- } \\
\text { minos topográficos que establecen distancia, proximidad, separación, } \\
\text { direccionalidad, etc. }\end{array}$ \\
\hline Recipiente & $\begin{array}{l}\text { Establecen límites físicos, pueden ser cuantificados, medidos, se em- } \\
\text { plean figuras geométricas o formas conocidas, tienen interior y exte- } \\
\text { rior. }\end{array}$ \\
\hline Personificación & $\begin{array}{l}\text { Se le atribuyen rasgos humanos a algo que no lo es, como motivacio- } \\
\text { nes, objetivos, acciones, características, etc. }\end{array}$ \\
\hline
\end{tabular}

A partir de esta tipología, se identificaron fragmentos en el discurso de construcción científica de los alumnos, en el que se usaron metáforas para explicar el sistema circulatorio del ser vivo, y se clasificaron de acuerdo a los tres tipos de metáforas empíricas.

Se presentan en este artículo algunos fragmentos discursivos (viñetas) usando nombres ficticios de los alumnos que participaron para respetar la confidencialidad del estudio, la intención es ejemplificar y, con ello, caracterizar los procesos de modelización científica de acuerdo a los tipos de metáfora de Lakoff y Johnson (1995).

\section{Resultados}

En el tema "¿Dónde están las venas?" Se encontraron en total 36 fragmentos discursivos en los que usaron metáforas empíricas, 4 del tipo orientacional, 14 de recipiente, 8 de personificación. También se pudieron identificar algunas combinaciones, entre ellas, 7 orientacionales y de recipiente, 2 orientacionales y de personificación y 1 de personificación y recipiente.

\section{Tipo orientacional}

Se identificaron cuatro diálogos en los que se emplearon metáforas de tipo orientacional, uno de ellos se muestra en la siguiente viñeta:

103. Maestra (00:11:43): ¿En los huesos qué? Dice Álvaro que por los huesos se sube.

104. César: No por los huesos, no se sube.

105. Maestra: ¿Por qué no?

106. César (00:11:54): Los, los huesos no están en las venas.

El diálogo anterior fue parte del rescate de saberes previos en relación con las venas. Para este momento, los alumnos habían descrito el proceso de circulación sanguínea a través de las venas únicamente como un movimiento descendente; cuando la maestra los cuestiona sobre la existencia de un movimiento en otro sentido, se presentaron dos respuestas, la anterior es una de ellas. 
Este fragmento contiene una metáfora orientacional al incluir elementos descriptivos de equivalencia asociados con la ubicación espacial, referentes a un movimiento ascendente dentro del proceso de circulación sanguínea.

Tres características de metáfora de tipo orientacional se encontraron dentro de estos cuatro fragmentos, en dos ocasiones se mencionó el movimiento ascendente, primero a través de los huesos y luego por una vena. En una ocasión se habló del movimiento descendente al explicar que la sangre se resbala y cae. Finalmente, se desestimó la posibilidad de que la sangre subiera por los huesos al presentar la idea de separación de las venas y los huesos.

\section{Tipo recipiente}

Se encontraron 14 fragmentos discursivos en las explicaciones de los alumnos, donde emplean metáforas del tipo recipiente. En la siguiente viñeta se presenta un ejemplo de ellos:

24. Maestra (00:05:15): Es diferente ¿Por qué?

25. José Pablo: Porque no tiene la punta filosa.

26. Maestra: Una, la punta de la venita que se encontró José Pablo en la planta tenía como una punta filosa, una punta delgadita, pero la vena que se encontró Eduardo en el pollo ¿Cómo era, Eduardo?

27. Eduardo (00:05:43): Tenía un hoyito.

Esta interacción se registró al cierre del tema acerca de las venas. Cuando se hacía una comparación entre las nervaduras de las plantas y las venas de pollo observadas, estableciendo semejanzas y diferencias. En este caso, las metáforas que emplearon tienen relación con las características de tipo recipiente pues los alumnos hacen referencia a formas conocidas y a objetos que tienen interior.

En los 14 fragmentos identificados se encontraron elementos pertenecientes a tres características de metáforas de tipo recipiente:

a) Formas conocidas: hablaron de resbaladillas y toboganes en 6 ocasiones, para ilustrar cómo se "resbala" la sangre en movimiento descendente. De tubos y escaleras una vez cada uno, cuando intentaban construir explicaciones sobre el mismo proceso en dirección opuesta. Por otro lado, al describir la forma de las venas observadas tanto en plantas como en el corazón de pollo, establecieron la comparación con puntas filosas en dos ocasiones, y picos una vez.

b) Mediciones y tamaños: con la mayor frecuencia consideraron las venas gorditas en 7 ocasiones. Flaquitas y grandecitas las dijeron 2 veces cada una. Medianas y delgadas solo una vez, todas en referencia a las nervaduras y venas que exploraron. 
c) Elementos que tienen interior y exterior, al hacer mención en una ocasión que la vena de pollo tenía un hoyito.

\section{Tipo personificación}

Se identificaron ocho fragmentos discursivos que presentan metáforas de tipo personificación, una de ellas es la siguiente viñeta:

2. Camilo (00:06:49): La sangre circula por las venas.

3. Maestra: La sangre circula por las venas, pero ¿cómo?

4. Rodrigo: A través.

5. Maestra: A ver ¿cómo?

5. Rodrigo: A través.

6. Maestra: ¿A través de qué?

7. Camilo: De las venas.

8. Maestra: De las venas, pero quién me explica ¿cómo le hace la sangre para circular? ¿qué es circular?

9. José Pablo: Que se vaya por todo el cuerpo.

10. Maestra: Que se vaya por todo el cuerpo.

11. Camilo (00:07:52): Porque si deja de caminar nos morimos.

Este ejemplo pertenece al momento inicial del tema, cuando se rescata lo aprendido en "Experiencias con la sangre" (tabla 1), y se busca vincularlo con los elementos del nuevo tema "¿Dónde están las venas?" Aquí se muestran las primeras ideas para construir una explicación colectiva respecto al proceso de circulación sanguínea; se considera que la metáfora que menciona Camilo es de personificación al asignarle el rasgo humano referente a caminar.

Las expresiones metafóricas que los alumnos mencionaron para ilustrar sus explicaciones sobre el proceso de circulación sanguínea dan cuenta de dos rasgos humanos; por un lado, establecen la comparación con acciones como caminar y resbalarse en dos ocasiones cada una, viajar y moverse una vez, y desarrollan la idea de que las venas engordan o adelgazan de acuerdo a la cantidad de comida que se consume, lo cual podría asociarse a características humanas de personificación.

La siguiente tabla reúne las características y frecuencias encontradas en los datos analizados, divididas por tipo de metáfora. 
Tabla 3. Concentrado de características y frecuencias de metáforas empíricas

\begin{tabular}{|l|l|l|}
\hline Tipo & Características & Frecuencia \\
\hline \multirow{4}{*}{ Orientacional } & Movimiento ascendente & 2 \\
\cline { 2 - 3 } & Movimiento descendente & 1 \\
\cline { 2 - 3 } & Separación & 1 \\
\cline { 2 - 3 } & Subtotal & 4 \\
\hline \multirow{4}{*}{ Recipiente } & Formas conocidas & 12 \\
\cline { 2 - 3 } & Medición y tamaño & 13 \\
\cline { 2 - 3 } & Elementos con interior y exterior & 1 \\
\cline { 2 - 3 } & Subtotal & 26 \\
\hline \multirow{3}{*}{ Personificación } & Acciones humanas & 6 \\
\cline { 2 - 3 } & Características humanas & 2 \\
\cline { 2 - 3 } & Subtotal & 38 \\
\hline \multirow{2}{*}{ Total } & & 8 \\
\hline
\end{tabular}

En el concentrado se puede apreciar que las características más recurrentes corresponden a elementos de medición y tamaño, así como formas conocidas (13 y 12 respectivamente), las cuales pertenecen al tipo de metáfora de recipiente. A partir de lo anterior, puede considerarse que los alumnos lograron en mayor medida establecer relaciones de equivalencia al mencionar atributos físicos de objetos conocidos, seguido de la característica de acciones humanas del tipo personificación con una frecuencia de 6 metáforas, cuando buscaban explicar el proceso de circulación sanguínea equiparando el movimiento de la sangre con un cuerpo humano que se desplaza en variedad de formas.

Entre las características con menor frecuencia se encuentran características humanas del tipo personificación presentes en dos diálogos: al comparar el grosor de las venas con el de un cuerpo humano, y al desarrollar una explicación causal en función del alimento consumido.

De las tres características de metáfora orientacional, el de movimiento ascendente se presentó dos veces, mientras que movimiento descendente una. Estas equivalencias fueron desarrolladas para caracterizar el proceso de circulación sanguínea como un flujo de doble sentido. La idea de separación estuvo presente en una ocasión, y corresponde a un argumento que negaba la posibilidad de que la sangre subiera a través de los huesos, ya que aseguraron los alumnos que son elementos que no comparten el mismo espacio en el cuerpo.

Se registró solo un elemento con interior y exterior, que pertenece a las metáforas de tipo recipiente, como una caracterización que permitió describir la observación de una vena en relación con un aspecto superficial de un objeto.

Ahora bien, durante el análisis de datos se identificaron algunos fragmentos discursivos en los que se presentan metáforas cuyas características pertenecen a dos tipos diferentes. En 
los próximos apartados se describen los casos en los que ocurrieron, y se explica la vinculación entre características.

\section{Orientacional + Recipiente}

En 7 ocasiones se identificó una combinación entre dos o más características de tipo orientacional y recipiente, la siguiente viñeta es una de ellas:

59. Maestra (00:10:02): A ver, Camilo dice que son dos toboganes.

60. Camilo: Es que son dos tubos, son dos tubos pa'que circule la sangre.

61. Maestra: Son dos tubos para que circule la sangre.

62. Camilo: Uno, uno pa'que suba y uno pa'que baje.

63. Maestra (00:10:57): Uno, en uno sube y en el otro baja.

En este fragmento se aprecian dos características del tipo orientacional, en el que se habla de un doble sentido en el que se desplaza la sangre en combinación con formas conocidas; en este caso mencionan objetos como tubos y toboganes, que pertenece al tipo recipiente.

De los 6 fragmentos discursivos restantes, 3 de ellos incluyen formas conocidas (FC), como mangueras, tubos y toboganes, y los relacionan con movimiento descendente (MD), referentes a caer o resbalarse. Otros 3, también abordan formas conocidas con movimiento ascendente (MA), al comparar el proceso de circulación cuando la sangre se encuentra en extremidades y partes del cuerpo superiores, con la acción de subir a la azotea usando escaleras o brincándose por una casa abandonada.

\section{Orientacional + Personificación}

Dos diálogos contienen características de tipo orientacional y personificación, uno es el que se presenta en la viñeta a continuación:

125. César (00:13:26): No, la sangre se va arrastrando para arriba.

126. Maestra: ¿La sangre qué?

127. César. La sangre se va arrastrando para arriba.

128. Maestra (00:13:45): La sangre se va arrastrando, dice César que la sangre se va arrastrando para arriba.

En ambos fragmentos se aprecia la característica de personificación, referente a la acción humana $(\mathrm{AH})$ de arrastrarse. En el primero (viñeta anterior), se menciona la acción únicamente y en el segundo se explica cómo se arrastraría la sangre si tuviera extremidades. Con respecto a 
la característica de metáfora tipo orientacional se trata de movimiento ascendente (MA) concerniente a la dirección en la que se desplaza.

\section{Personificación + Recipiente}

Solo se identificó un diálogo en el que se relacionan características de personificación con recipiente y se encuentra en la siguiente viñeta:

129. Carla (00:13:47): Se va agarrando, como una escalera.

130. Jesús: No tiene manos.

131. Maestra: Dice Jesús que no se puede arrastrar porque no tiene manos. ¿Entonces cómo se arrastra?

132. Jesús (00:14:08): Como un gusano.

Se aprecian tres características en este caso, una de formas conocidas (FC) perteneciente a metáfora de tipo recipiente, al mencionar la escalera como elemento explicativo para ilustrar un proceso, y dos de personificación referentes a la acción humana (AH) de arrastrase para caracterizar el movimiento de la sangre durante la circulación sanguínea, y a la característica humana $(\mathrm{CH})$ de manos, elemento que sirve de argumento para negar la posibilidad de usar una escalera.

En la siguiente tabla se muestran las características encontradas y su frecuencia en los casos en los que se vincularon tipos de metáfora empírica.

Tabla 4. Concentrado de características y frecuencias de combinaciones de metáforas empíricas

\begin{tabular}{|l|r|r|r|r|r|c|}
\hline \multirow{2}{*}{$\begin{array}{c}\text { Combinaciones de tipo de me- } \\
\text { táforas empíricas }\end{array}$} & \multicolumn{4}{|c|}{ Características } & $\begin{array}{c}\text { Cantidad de } \\
\text { fragmentos } \\
\text { discursivos }\end{array}$ \\
\cline { 2 - 7 } & $\mathrm{MA}$ & $\mathrm{MD}$ & $\mathrm{FC}$ & $\mathrm{AH}$ & $\mathrm{CH}$ & \multicolumn{1}{c|}{\begin{tabular}{l}
$\mathrm{F}$ \\
\hline Orientacional + Recipiente
\end{tabular}} \\
\hline Orientacional + Personificación & 2 & - & - & 2 & - & 2 \\
\hline Personificación + Recipiente & - & - & 1 & 1 & 1 & 1 \\
\hline Total & 6 & 4 & 8 & 3 & 1 & 10 \\
\hline
\end{tabular}

La tabla anterior permite reconocer que la característica más empleada por los alumnos durante sus explicaciones, en las que combinan distintos tipos de metáfora, son las formas conocidas $(\mathrm{FC})$ con una frecuencia de 8 , es decir, de los 10 diálogos en los que usaron metáforas en vinculación, solo en 2 no hicieron uso del recurso de nombrar objetos que encuentran en su contexto. 
Otro dato que se observa en el concentrado es que en 10 ocasiones trataron de explicar el proceso de circulación sanguínea mediante el recurso topográfico de la direccionalidad, 6 de estos desarrollan la idea de movimiento ascendente (MA) y 4 la de movimiento descendente (MD). Las anteriores son las características con más registros, con ello se puede entender que los alumnos de preescolar usan metáforas relacionadas con elementos físicos y direccionalidad en mayor medida, al caracterizar procesos como la circulación sanguínea.

Las metáforas con menor frecuencia corresponden al tipo de personificación, acciones humanas $(\mathrm{AH})$ y características humanas $(\mathrm{CH})$ que usan como estrategia para describir la labor específica de la sangre comparándola con un cuerpo.

En el siguiente apartado se presenta el modelo teórico escolar que los alumnos construyeron durante el tema "¿Dónde están las venas?", y se describen sus elementos a partir de las metáforas que emplearon en los discursos explicativos.

\section{Modelo teórico escolar}

Como resultado del proceso de análisis discursivo y en contraste con el modelo teórico de sistema circulatorio del cuerpo humano de referencia (figura 1), se concentraron los significados consensuados por el grupo de estudiantes de preescolar y se conformó un modelo teórico escolar.

Dicho modelo se fue enriqueciendo a lo largo de cinco temas de trabajo (tabla 1). En este artículo se le llama modelo teórico escolar en construcción (figura 2) debido a que únicamente se incluyen los significados e ideas desarrolladas durante el tema “¿Dónde están las venas?". En este modelo se aprecian algunos elementos de la manera en que fueron expresados, respetando las metáforas empleadas. 
Figura 2. Modelo teórico escolar en construcción

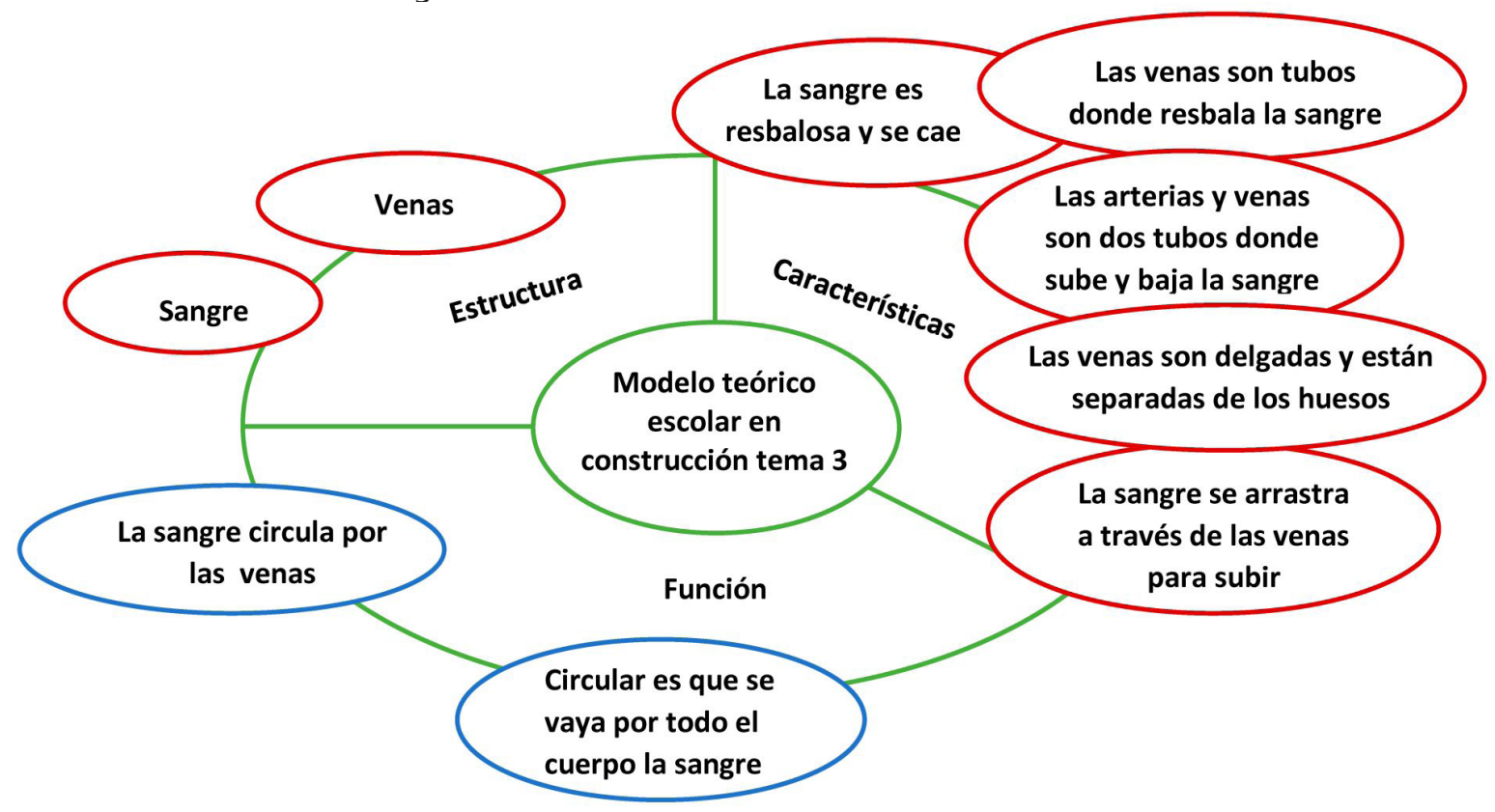

De acuerdo con el modelo teórico de referencia (figura 1), los elementos de estructura que componen el sistema circulatorio son el corazón, la sangre, las venas y las arterias, y cada uno posee cualidades específicas. En el modelo teórico escolar en construcción se mencionan solo dos elementos (sangre y venas).

Con respecto a la función genérica del sistema circulatorio, de acuerdo a Gutiérrez (2015), se trata de bombear sangre oxigenada desde el corazón hacia el resto de órganos y enviar de regreso la sangre no oxigenada por medio de venas y arterias. Por su parte, los niños construyeron dos ideas interrelacionadas: la sangre es el elemento que circula a través de las venas y dicha acción implica moverse por todo el cuerpo.

En el modelo teórico de referencia (figura 1) se presenta el elemento de enfermedad; sin embargo, en este caso no se abordó, lo que sí se rescató y se consideró importante integrar al modelo teórico escolar, fueron las metáforas usadas como recurso explicativo a lo largo del tema “¿Dónde están las venas?”. En el modelo construido por los estudiantes, las llamamos características ya que permitieron ilustrar de manera más clara las descripciones colectivas.

Desde la clasificación de Lakoff y Johnson (1995), el tipo de metáfora de recipiente se considera el más empleado. Las dos características que registraron mayor frecuencia fueron, formas conocidas $33.3 \%$ y medición y tamaño $21.7 \%$; la característica de elementos con interior y exterior se presentó en $1.7 \%$, no figura en el modelo puesto que se trató de la descripción de una observación durante la experimentación. 
El segundo tipo de metáfora con mayor frecuencia en las explicaciones y que permite conformar el modelo es el orientacional. Al respecto, se observa que predominan las ideas sobre una doble direccionalidad en la que se mueve la sangre, es decir, no toman como punto de referencia el corazón como en el modelo teórico (figura 1), sino la idea de subir o bajar.

Las oraciones relacionadas con movimiento ascendente comprenden 13.3\%, mientras que movimiento descendente constituye $8.3 \%$. La idea de que las venas y huesos ocupan espacios distintos dentro del cuerpo (separación), solo registró 1.7\%. Sin embargo, fue aceptada por todos y por eso se presenta en el modelo teórico escolar en construcción.

El tipo de metáfora con menor presencia en los discursos explicativos es de personificación, los alumnos desarrollan las ideas de caer y arrastrarse (acciones humanas) en 15\% y describen las venas con adjetivos calificativos como delgadas o flaquitas (características humanas) $5 \%$ de las veces.

\section{Una mirada a la intervención docente}

Un punto que es importante considerar, es la influencia de la maestra como elemento generador de metáforas en el discurso explicativo del alumno. En relación con las metáforas de tipo orientacional específicamente, es donde se aprecia la influencia de la maestra al introducir la idea del movimiento ascendente.

Como se describe al inicio del apartado de resultados, durante las explicaciones iniciales de los niños con respecto al proceso de circulación, ellos hacían referencia solo a un movimiento descendente.

Se observa en los datos que la intervención docente con preguntas que cuestionaban, por qué la sangre solo se desplaza en una dirección, fue el elemento que condujo a la caracterización del proceso de circulación en un doble sentido.

Por tanto, se podría considerar, de acuerdo con la proporción de metáforas que configuran el modelo teórico en construcción, que $13.3 \%$ correspondiente a las oraciones relacionadas con movimiento ascendente fueron una elaboración colectiva, en conjunto con la maestra, mientras que $86.7 \%$ restante son significados consensuados únicamente entre alumnos.

\section{Discusión y conclusiones}

Se ha documentado el creciente interés por conocer cómo aprenden ciencias los alumnos de preescolar en México (Gómez et al., 2013); así también, se han implementado con éxito propuestas de secuencias didácticas basadas en la experimentación a través de los sentidos (CanedoIbarra et al., 2012; Gómez, 2007). El presente trabajo aporta a esta tendencia la caracterización de un recurso explicativo que se encontró en el discurso científico de los estudiantes de este nivel educativo: las metáforas. 
En relación con el objetivo de investigación, referente a caracterizar los procesos de modelización científica al elaborar un modelo teórico escolar de ser vivo enfocado al sistema circulatorio, los resultados muestran que uno de esos procesos corresponde al uso de metáforas empíricas.

Para Wigdorovitz (2014), representan una herramienta mental que configura los saberes, porque amplían las posibilidades de expresión y, al mismo tiempo, establecen un marco de construcción de significados. En este caso, los estudiantes hicieron uso de metáforas con las que delimitaron conceptualmente el proceso de circulación sanguínea, así como los órganos involucrados, a partir de establecer relaciones con elementos de su contexto que tenían sentido para ellos.

Desde Lakoff y Johnson (1995), las metáforas empíricas permiten organizar conceptos en un sentido global basado en la experiencia física y tomando como punto de referencia el propio cuerpo, esto es, estableciendo vínculos entre el fenómeno y sus vivencias.

Si bien el uso de metáforas permite modificar algunas de las concepciones iniciales (Canedo-lbarra et al., 2012) de los alumnos de preescolar, con respecto a un fenómeno del ser vivo como es la circulación sanguínea, no podríamos hablar de un proceso de abstracción en el sentido estricto del término, el cual es un elemento característico del pensamiento científico en edades posteriores.

Lo que se confirma en este trabajo es que los estudiantes vincularon sus experiencias previas con el modelo teórico de referencia, mediante el recurso explicativo de las metáforas empíricas y, a partir de ello, establecieron delimitaciones conceptuales. En este caso, las metáforas empíricas influyeron directamente en la caracterización del proceso de circulación y los órganos involucrados, como se explica a continuación.

La delimitación conceptual que se aprecia a partir de las metáforas de tipo recipiente, tiene que ver con la caracterización de los elementos del modelo teórico escolar en construcción (figura 2), es decir, la relación de semejanza entre las venas con objetos que encuentran en su contexto como son los tubos, es aceptada por todos los alumnos.

Por otra parte, las metáforas de tipo orientacional sirvieron para ilustrar el flujo de la sangre en dos sentidos a lo largo del cuerpo humano. Finalmente, las metáforas de personificación aportaron algunas explicaciones de funcionamiento referentes a acciones que realiza la sangre durante su recorrido, en similitud con un cuerpo humano.

Si bien la intervención docente es un factor que configura los procesos de modificación de concepciones iniciales de la ciencia en el ámbito escolar, es posible considerar en este caso específico, que la influencia de la maestra como agente generador de metáforas fue determinante solo en las de tipo orientacional, las cuales constituyen menos de la quinta parte de las caracterizaciones del modelo teórico escolar. 
Por lo tanto, como se puede apreciar en este trabajo, los alumnos de preescolar son capaces de construir, en interacción con sus pares, significados consensuados en torno al sistema circulatorio del ser vivo, con recursos explicativos como las metáforas.

Con respecto al tipo de modelo que construyen los estudiantes de preescolar, se toma la clasificación del trabajo de Saçkes (2015), en el que desarrollaron tres tipos de modelos mentales al hablar y hacer ciencia: inicial, sintético y científico. En el caso del modelo teórico escolar en construcción, cuenta con características de modelo sintético, ya que nombra los elementos, los describe y establece relaciones entre ellos; con lo cual es posible comprender sus ideas en torno al proceso de circulación.

Es evidente en este caso, la función mediadora de potencia analógica que los alumnos desarrollaron a través del discurso. Para Adúriz-Bravo et al. (2005), los mediadores tienen un alcance restringido y contextual. Sirven para dar sentido a la ciencia que se hace en la escuela dentro del espacio de experimentación y, durante la construcción de explicaciones, son modelos de segundo orden que permiten integrar ideas al modelo teórico escolar.

Por lo anterior y en contraste con los resultados, es posible asegurar que los alumnos delimitaron el código, favoreciendo la construcción de significados en torno al sistema circulatorio a través del empleo del recurso explicativo de metáforas empíricas, pues establecieron relaciones de semejanza con su espacio físico y con su cuerpo.

Por lo tanto, se comparte el argumento de diversos estudios respecto a la pertinencia del enfoque de modelización al hacer ciencia en el aula preescolar (Canedo-lbarra et al., 2012; Mazas et al., 2018). Este enfoque permite ampliar y mejorar los conocimientos en torno a la ciencia.

Se agrega, además, que enfocarse en el discurso, en especial en recursos explicativos como las metáforas, favorece la compresión de las ideas de los estudiantes y el desarrollo de habilidades que acerquen al niño en edad preescolar al pensamiento científico. Finalmente, se considera necesario seguir fortaleciendo la línea de investigación sobre modelización e indagar la variedad de recursos para construir significados.

\section{Referencias}

Adúriz-Bravo, A., A. Gómez, C. Márquez y N. Sanmartí (2005) La mediación analógica en la ciencia escolar. Propuesta de la "función modelo teórico". Enseñanza de las Ciencias, Número Extra. VII Congreso.

Acher, A., M. Arcà y N. Sanmartí (2007). Modeling as a Teaching Learning Process for Understanding Materials: A Case Study in Primary Education. Sciencie Education, 91, 398-418. DOI: $10.1002 /$ sce.20196

Ahi, B. (2016). A Study to Determine the Mental Models in Preschool Children's Conceptualization of a Desert Environment. International Electronic Journal of Elementary Education, 8(3), 333-350. 
Candela, A. (1999). Prácticas discursivas en el aula y calidad educativa. Revista Mexicana de Investigación Educativa, 4(8), 273-298.

Canedo-Ibarra, S., J. Castelló-Escandell, A. García-Wehrle, A. Gómez-Galindo y A. Morales-Blake (2012). Cambio conceptual y construcción de modelos científicos precursores en educación infantil. Revista Mexicana de Investigación Educativa, 17(54), 691-727.

Cruz-Guzmán, M., A. García y A. M. Criado (2017). Aprendiendo sobre los cambios de estado en educación infantil mediante secuencias de pregunta-predicción-comprobación experimental. Enseñanza de las ciencias, 35(3), 175-193. https://doi.org/10.5565/rev/ensciencias. 2336

Espinet, M., M. Izquierdo, J. Bonil y L. Ramos (2012). The Role of Language in Modeling the Natural World: Perspectives in Science Education. En Fraser, B. T. (ed.). Second International Handbook of Science Education. Nueva York: Springer, 1385-1403.

García, M.P. (2005). Los modelos como organizadores del currículo en biología. Enseñanza de las Ciencias, Número Extra, 1-6.

Giere, R. (1988). Explaining Science: A Cognitive Approach. Chicago: Chicago University Press.

Gómez, A. (2005). La construcción de un modelo de ser vivo en la escuela primaria: Una visión escalar. (Tesis doctoral). Universitat Autònoma de Barcelona, Barcelona (inédita).

(2007). Los seres vivos y su relación con el medio: propuesta para abordar el modelo "relación" en la escuela primaria. http://www.manosalaobra.cinvestav.mx/pdf/biblioteca/invest/ enseñanza/funcion\%20relacion\%20primaria.pdf

(2009). El estudio de los seres vivos en la educación básica: Enseñanza del sistema nervioso desde un enfoque para la evolución de los modelos escolares. Monterrey: Universidad Autónoma de Nuevo León.

Gómez-G., A., F. A. García y G. C. M. García (2013). Estado de la Investigación en Educación en Ciencias Naturales en el Nivel de Educación Básica, durante la década 2002-2012. En Ávila, A., A. Carrasco, A. Gómez et al. (coords.). Una década de investigación educativa en conocimientos disciplinares en México. Matemáticas, ciencias naturales, lenguaje y lenguas extranjeras. México: COMIE-ANUIES.

Gómez-Motilla, C. y J. Ruiz-Gallardo (2016). El rincón de la ciencia y la actitud hacia las ciencias en educación infantil. Revista Eureka sobre Enseñanza y Divulgación de las Ciencias, 13(3), 643-666. http://dx.doi.org/10.25267/Rev Eureka ensen divulg cienc.2016.v13.i3.10

Gutiérrez, L. (2015). Modelos y modelización de sistemas del cuerpo humano. Estudio de caso de una maestra de cuarto grado de primaria. (Tesis doctoral). Universidad Pedagógica Nacional, Bogotá, Colombia (inédita).

Howitt, C., S. Lewis y E. Upson (2011). 'It's a mystery!' A Case Study of Implementing Forensic Science in Preschool as Scientific Inquiry. Australasian Journal of Early Childhood, 36(3), 45-55. 
Izquierdo, M., M. P. Espinet, R. Pujol y N. Sanmartí (1999). Caracterización y fundamentación de la ciencia escolar. Revista Enseñanza de las Ciencias, número extra, 79-91.

Lakoff, G. y M. Johnson (1995). Metáforas en la vida cotidiana. Madrid: Ediciones Cátedra.

Lemke, J. (1990). Talking Science: Language, Learning and Values. Norwood: Ablex.

Mazas, B., J. Gil-Quílez, B. Martínez, A. Hervas y A. Muñoz (2018). Los niños y las niñas de infantil piensan, actúan y hablan sobre el comportamiento del aire y del agua. Enseñanza de las Ciencias, 36(1), 163-180. https://doi.org/10.5565/rev/ensciencias.2320

Oğuzhan A., M. Altan, y B. Ahí (2013). Mental Models of School for Preschool Children. European Journal of Educational Research, 2(2), 97-105.

Saçkes, M. (2015). Kindergartners' Mental Models of the Day and Night Cycle: Implications for Instructional Practices in Early Childhood Classroom. Educational Sciences: Theory \& Practice, 15(4), 997-1006. DOI: 10.12738/estp.2015.4.2741

Secretaría de Educación Pública (2012). Programa de Estudios 2011. México: SEP.

Wigdorovitz, A.R. (2014) Las metáforas conceptuales en la construcción del discurso pedagógico. Revista de Educación, 5(7), 17-32. 